Ann. Zootech., I976, 25 (2), I89-197.

\title{
L'ALIMENTATION AZOTÉE DU CANETON DE BARBARIE : ÉTUDE DU BESOIN DU CANETON MÂLE ENTRE LES ÂGES DE 4 ET 12 SEMAINES
}

\author{
B. LECLERCQ et H. DE CARVILLE \\ Station de Recherches avicoles, \\ Centre de Recherches de Tours, I. N.R. A., \\ Nouzilly, 37380 Monnaie, B.P. 1
}

\begin{abstract}
RÉSUMÉ
Des canetons mâles de Barbarie sont élevés jusqu'à l'âge de 4 semaines avec un même aliment satisfaisant largement leurs besoins. On leur distribue ensuite des régimes isoénergétiques ( I 2,6 MJ EM/ $/ \mathrm{kg}$ ) ne différant que par leur teneur en protéines : Io,6, I2, I 3,4, 14,9 et 16,3 p. Ioo.

Le besoin en protéines (régimes à base de maïs et de tourteau de soja) du caneton mâle n'cst pas différent de celui de la femelle et n'excède pas I 5 p. Ioo entre 4 et 8 semaines d'âge, I 2 p. roo entre 8 et ro scmaines. Au-delà, il devient inférieur aux valeurs de la gamme retenue dans notre essai.

La dissection des animaux sacrifiés aux âges de 74 et 88 jours montre que la cuisse atteint son poids maximum à l'âge de 74 jours. Le filet, au contraire, s'accroît entre ces deux stades en valeur absolue et en valeur relative. La proportion de filet (en pourcentage du poids vif) ne dépend pas du poids de l'animal mais essenticllement de son âge.
\end{abstract}

\section{INTRODUCTION}

Une première étude nous a permis de préciser le besoin en protéines du Caneton femelle de Barbarie entre les âges de 4 et i I semaines (LECLERCQ et de CARVILLE, I975). Avec des régimes à base de maïs et de tourteau de soja et titrant $12,5 \mathrm{MJ} / \mathrm{kg}$ (3000 kcal $/ \mathrm{kg}$ ) d'énergie métabolisable le besoin ne dépasse pas I $5 \mathrm{p}$. Ioo entre les âges de 4 et 6 semaines et devient inférieur à $\mathrm{I} 3$ p. Ioo au-delà. Une nouvelle étude a été entreprise sur le Caneton mâle dont la vitesse de croissance est beaucoup plus élevée que celle de la femelle. C'est l'objet de la présente publication. Comme dans le cas de la femelle, nous avons réalisé des dissections afin d'apprécier l'évolution des parties comestibles en fonction de l'âge et des caractéristiques du régime. 


\section{MATÉRIEL E'T MÉTHODES}

I'our ce qui concerne les animaux et les conditions d'élevage, nous avons recours aux méthodes précédemment décrites (LeCLERCQ et de CARVILLE, I975), si ce n'est que nous utilisons des canetons mâles âgés de 3 jours au début de l'essai.

Tous les animaux reçoivent pendant les 4 premières semainos de vie un même régime de démarrage complet et équilibré dont la composition est présentée dans le tableau $\mathbf{I}$. Par la suite, on constitue 5 lots de 20 animaux (2 répétitions de Io) recevant chacun un des régimes expérimentaux. La clénomination de ces lots correspond à la teneur théorique en matières azotées totales (MAT) du régime distribué : I2, I3, I f, I5, I6. Ces régimes sont obtenus par mélange des deux régimes I 2 et 16 dont les compositions sont rapportées dans le tableau I. La détermination des tencurs réelles en . VAT après analyse par la méthode de Kjeldahl est la suivante:

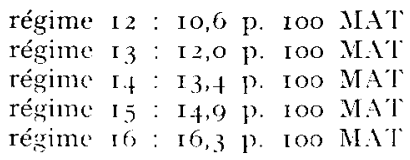

TABI IEAU I

Composition des regimes

Régine de Régine 16 R Réginte 1:
Démarrage

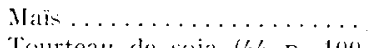

Mateal de soja (1́t p. 100

MAT) $\ldots \ldots \ldots \ldots \ldots \ldots$

Huile d'arachide ..........

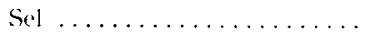

Carbonate de calcium ......

I'hosphate bicalcique ......

Oligo-minéraux .........

Málange vitaminique ......

I)]-méthionine ..........

\begin{tabular}{|c|c|c|}
\hline 59 & fist & 87 \\
\hline $3 \overline{3}$ & 26 & $x$ \\
\hline 1 & $\because$ & 1 \\
\hline 0,3 & $11,: ;$ & $11,: ;$ \\
\hline 1 & 1 & 1 \\
\hline$\ddot{9}$ & $\because$ & $\because$ \\
\hline 0,1 & $(1,1$ & 0,1 \\
\hline 1,6 & 1,$1 ;$ & 1,6 \\
\hline 0,1 , & $11,1: 32$ & \\
\hline
\end{tabular}

Tallx énergeticule calculé (cinergie mótabolisable) :

\begin{tabular}{|c|c|c|c|}
\hline $\mathrm{NJ} / \mathrm{k}_{\mathrm{m}} \ldots \ldots \ldots \ldots \ldots$ & $11,9.5$ & $12, x()$ & 12,9111 \\
\hline Kical/kg ........... & 29611 & 30.50 & $\because$ afis \\
\hline en MAT mesuree $(\%)$. & $2 \geq, 11$ & 16,3 & 111,6 \\
\hline
\end{tabular}

Tenemer en MAT mesuree $(\%)$. 20,0 16,3

Composition du mélange oligo-minéral (p. $1100 \mathrm{~kg}$ ) oxyde de mangancse : 7 , oxyde de zinc: $5 g$, sulfate ferreux : $0,55 \mathrm{~g}$, carbonate de cuivre: $10,0,3 \mathrm{~g}$ iodure de potassium : $11,1 \mathrm{~g}$, carbonate de cobalt : $0,03 \mathrm{~g}$, carbonate de calrilu11: $80,5 \mathrm{~g}$.

Composition du mélange ritaminiqu': vitamine A : xom $1100 \mathrm{C}$, vitamine $\mathrm{I}_{3}: 100000 \mathrm{C}$, vitamine $\mathrm{K}: 0, \underline{\mathrm{g}} \mathrm{g}$, riboflavine : $: 2 \mathrm{~g}$, acide nicotinique: $5 \mathrm{~s}$, accicle folique : $0,6 \mathrm{~g}$, pyridoxine : $0,6 \mathrm{~g}$, biotine : $0,1 \mathrm{~g}$, cyanocobalamine : 0,5 ng, pantothénate de calcium : 2 2 , choline : $150 \mathrm{~m}$. 
les animaux idcntifiés par une bague métallique fixée à l'aile sont pesés individucllement à jeun aux âges de $5,6,7,8,9$, I0, I I et I 2 semaincs. La consommation d'aliment est enregistrée aux mêmes stades pour chaque répétition.

Aux âges de ro semaines et + jours ( 74 jours) et de 12 semaines et + jours (88 jours) la moitié de chaque répétition est sacrifiée. Les oiscaux sont saignés et plumés. On procède ensuite à la disscction selon la méthode décrite précédemment (LECLERcQ et de CARviLle, I97.5).

\section{RÉSULTATS}

Le tableau 2 renferme les valeurs moyennes du poids vif des animaux en fonction de l'âge et du régime distribué. Les calculs statistiques sont réalisés sur les gains de poids individuels dont les valeurs moyennes figurent dans le tableau 3. Jusqu'à l'âge de Io semaines, la croissance du lot $\mathrm{I} 2$ (régime renfermant ro,6 p. Ioo de MAT) est toujours plus faible que celle des autres lots. A l'opposé, les lots I 5 et I 6 présentent des gains de poids plus élevés que les autres de 4 à 6 semaines. Entre 6 et 8 semaines, le gain de poids du lot I4 atteint une valeur aussi élevée que les lots recevant les régimes les plus riches. Puis de 8 à ro semaines, le régime I 3 permet le même gain de poids que les régimes mieux pourvus en protéines.

Les différences observées dans les résultats de consommation alimentaire sont moins accusées. La teneur en protéines des régimes ne modifie guère cette consomma tion sauf dans le cas de carence importante. Les excès de protéines ne semblent pas influencer le niveau d'ingestion d'aliment. L'indice de consommation prend des valeurs de plus en plus élevées à mesure que les animaux vieillissent surtout après l'âge de ro semaines. Les différences entre les lots reflètent assez fidèlement les différences induites par les régimes sur la croissance pondérale.

Les résultats des dissections réalisées aux âges de 74 et 88 jours sont présentés dans le tableau 5. Le rendement à l'abattage n'est influencé ni par les régimes ni par l'âge des animaux, ni par leur poids vif (corrélation de o,2I non significative). Le poids du filet s'accroît avec l'âge d'abattage et il est sous la dépendance du régime distribué ; les régimes I2 et I 3 ne permettent pas aux canetons de développer leurs masses musculaires pectorales. En fait l'analyse de ces résultats nécessite le recours à l'analyse de covariance afin de savoir d'une part si le rapport $\frac{\text { poids du filet }}{\text { poids vif }}$ dépend des régimes distribués et d'autre part s'il est lié à l'âge de l'animal ou à son poids vif. C'est l'objet des tableaux 6 et 7 . Dans le tableau 6 nous rapportons les résultats d'analyse de covariance en fonction des régimes à chacun des stades d'abattage. L ors du premier abattage, on ne met en évidence aucun effet spécifique des régimes: les filets représentent toujours la même proportion du poids vif quel que soit le lot expérimental. A 88 jours, on enregistre un effet significatif du régime révélant que la proportion de filet est inférieure dans les deux lots recevant le moins de protéines. L'influence de l'âge d'abattage fait l'objet du tableau 7. Nous ne retenons pour les calculs que les lots ayant présenté des vitesses de croissances très voisines. On voit qu'indépendamment de l'effet de l'âge sur le poids vif, l'âge d'abattage exerce un effet spécifique sur la taille des filets : à 88 jours ceux-ci représentent une part plus importante du poids vif qu'à 74 jours. C'est ce qu'illustre la figure I. On troure aussi dans le tableau 7 les deux équations de régression linéaire reliant le poids du filet 


\section{TABLEAU 2}

Évolution du gain de poids vif (en g) des canetons de Barbarie (mâles)

\begin{tabular}{|c|c|c|c|c|c|}
\hline Lot & 12 & 13 & $1 / 1$ & 15 & 16 \\
\hline $\begin{array}{c}\text { Teneur en MAT } \\
(\%)\end{array}$ & 10,6 & $1 \geq, 0$ & 13,4 & 11,9 & 16,3 \\
\hline Age (semaines) : & & & & & \\
\hline 4 & 1089 & 1084 & 1098 & 1108 & 1116 \\
\hline 5 & 1489 & 1576 & 1586 & 1626 & $165:$ \\
\hline 6 & 1807 & 2050 & 2108 & 2194 & $2 \geq 11$ \\
\hline 7 & 2119 & 2415 & 2585 & 2701 & 2715 \\
\hline 8 & 2280 & 2639 & 2878 & 3030 & $30 \times 1$ \\
\hline 9 & 2504 & 2923 & 3199 & 3359 & 3376 \\
\hline 10 & 2687 & 3162 & 3453 & 3591 & 3610 \\
\hline $11(1)$ & 2882 & 3347 & 3589 & 3641 & 3782 \\
\hline $12\left(^{1}\right)$ & 3039 & 3539 & 3730 & 3745 & 3853 \\
\hline
\end{tabular}

(1) A ces stades, chaque lot ne contient plus que la moitié de l'effectif, l'autre moitié ayant été sacrifiée à l'âge de 10 semaines et 1 jours.

\section{TABLEAU 3}

Évolution du poids vif (en g) des canetons de Barbarie (mâles)

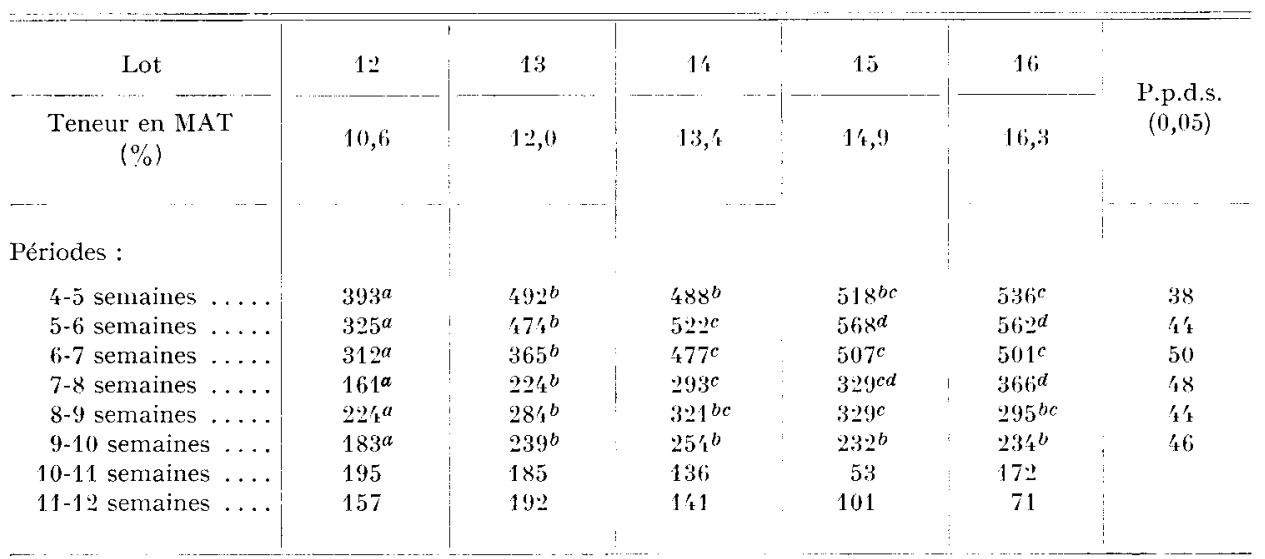

Note: les moyennes accompagnées d'une même lettre ne sont pas significativement différentes au seuil $(\mathrm{P}=0,05)$. 
Sivolution de la consommation d'aliment (C) (en g)

et de l'indice partiel de consommation (I) des canetons de Barbarie (mâles)

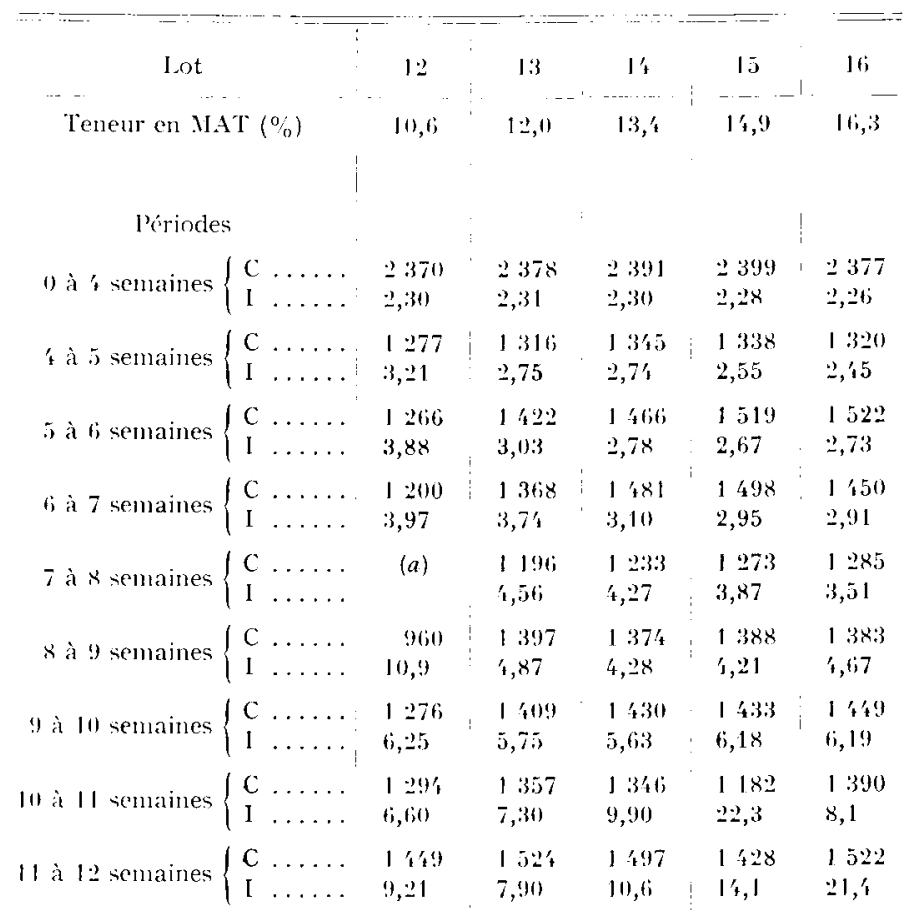

(a) Wortalité importante par cannibalisme.

\section{TABLEAU 5}

Rendement à l'abattage, poids des filets et des cuisses en fonction des régimes

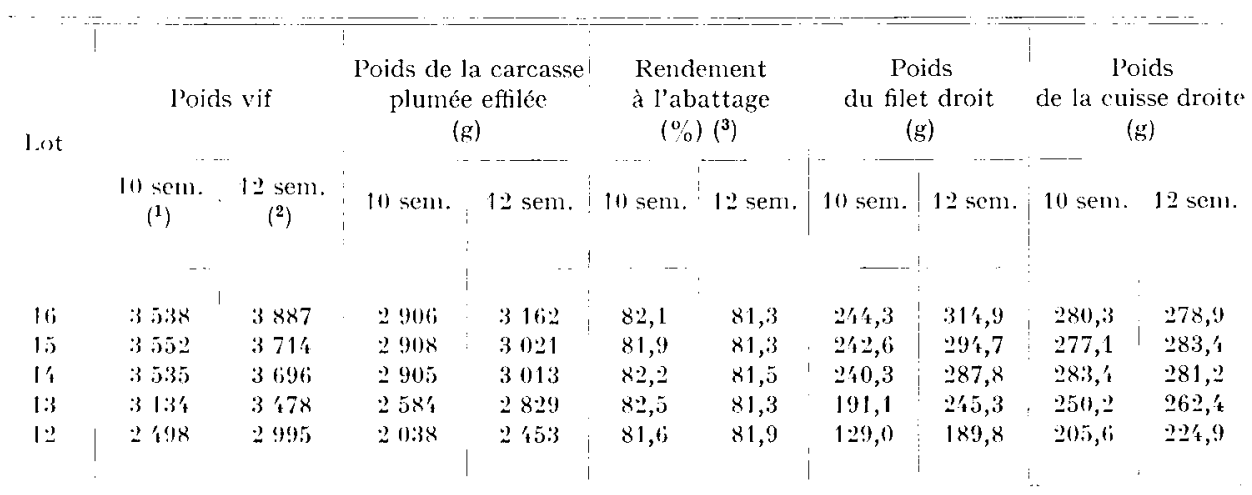

(1) En réalité 10 semaines et ' jours.

(2) En réalité 12 semaines et 1 jours.

(3) Rapport du poids de la carcasse plumée et effilée sur le poids vif, multiplić par 100. 
Résultats de l'analyse de covariance (valeurs ajustées)

entre le poids du filet ou de la cuisse droite

et le poids vif du Caneton male de Barbavie

en fonction du taux protidique du régime (ensemble des lots)

1) Filct.

Corrélation générale

Test dc non parallelisme

valeur de $\mathrm{F} \ldots \ldots \ldots \ldots \ldots \ldots$

degrés de liberté ...........

liffet du regime

valeur de $\mathrm{F} \ldots \ldots \ldots \ldots \ldots \ldots \ldots$

degrés de liberté

Différence entre lots (1)

2) Cuisse.

Corrélation générale

Test de non parallélisme

valeur de $F \ldots \ldots \ldots \ldots \ldots \ldots \ldots$
degrés de liberté $\ldots \ldots \ldots \ldots \ldots$

$r=-11,853^{* *}$

degrós de lib

valeur de $F \ldots \ldots \ldots \ldots \ldots$.

degrés de liberté $\ldots \ldots \ldots \ldots \ldots$ $r=+0,619 * *$

$$
r=-0,761 * *
$$

$$
0,7 \times \text { NS }
$$

í et 3 ?

$1,63 \mathrm{NS}$

bet $3 s$

$$
\begin{aligned}
& 0,17 \times S \\
& \text { i et } 3 / 4
\end{aligned}
$$

(0,5) NS

's et $3{ }^{\prime}$

0,16 NS

set 38
$1,75 \mathrm{NS}$

fet 38

$$
r=\div \quad+0,78: 3^{* *}
$$

$0,711 \mathrm{NS}$

4 et 3 ?

$i, i)(1 * *$

4 ct $3 \mathrm{~S}$

$1 \cdot u$

1,3,

$1, b$

$15^{b}$

$16^{2}$

** Effet significatif au seuil $P=0,01$.

(1) Les lots suivis de la même lettre ne sont pas significativement différents $(P=0,15)$.

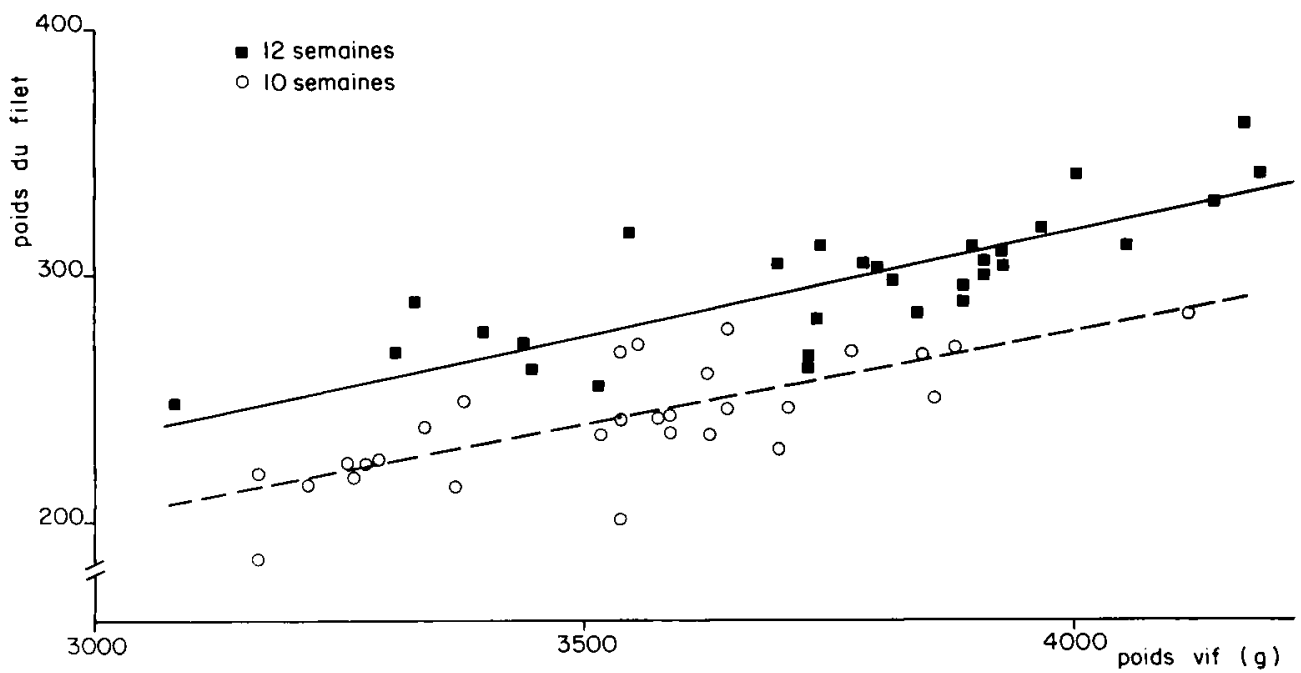

lis. r. - Evolution du poids du flet en fonction du poids vif chez le Caneton de Barbarie mâle 
Résultats de l'analyse de covariance (valeurs ajustées) entre le poids du flet droit ou de la cuisse droite

et le poids vif du Caneton male de Barbarie

en fonction de l'áge d'abattage (animaux cles lots I, I, et I6)

Age d'abattage

1) Filet

Corrélation

Test de parallélisme

valeurs de $F^{*} \ldots \ldots \ldots \ldots \ldots \ldots$

degrés de liberté

liffet de l'âge

valeur de $1 ; \ldots \ldots \ldots \ldots \ldots \ldots$.

degrés de liberté .............

Equation de régression (1)

2) Cuise.

Corrélation

Test de non parallélisme

valeur de $\mathrm{F} \ldots \ldots \ldots \ldots \ldots \ldots$

degrés de liberté ............

liffet de l'âge

valeur de $1 . \ldots \ldots \ldots \ldots \ldots \ldots$.

degrés de liberté .............

Equation de régression $\left({ }^{1}\right)$

** Effet significatif au seuil $P=0,01$.

(1) $y==$ poids du filet ou de la cuisse en grammes,

$r=$ poids vif en grammes.

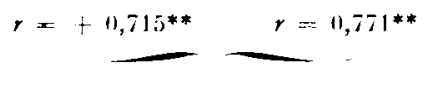

0,118 NS

1 et 56

$63,2 * *$

1 et 57

$y=0,11754 x-21,4 \quad y=11,0761 x+11,10$

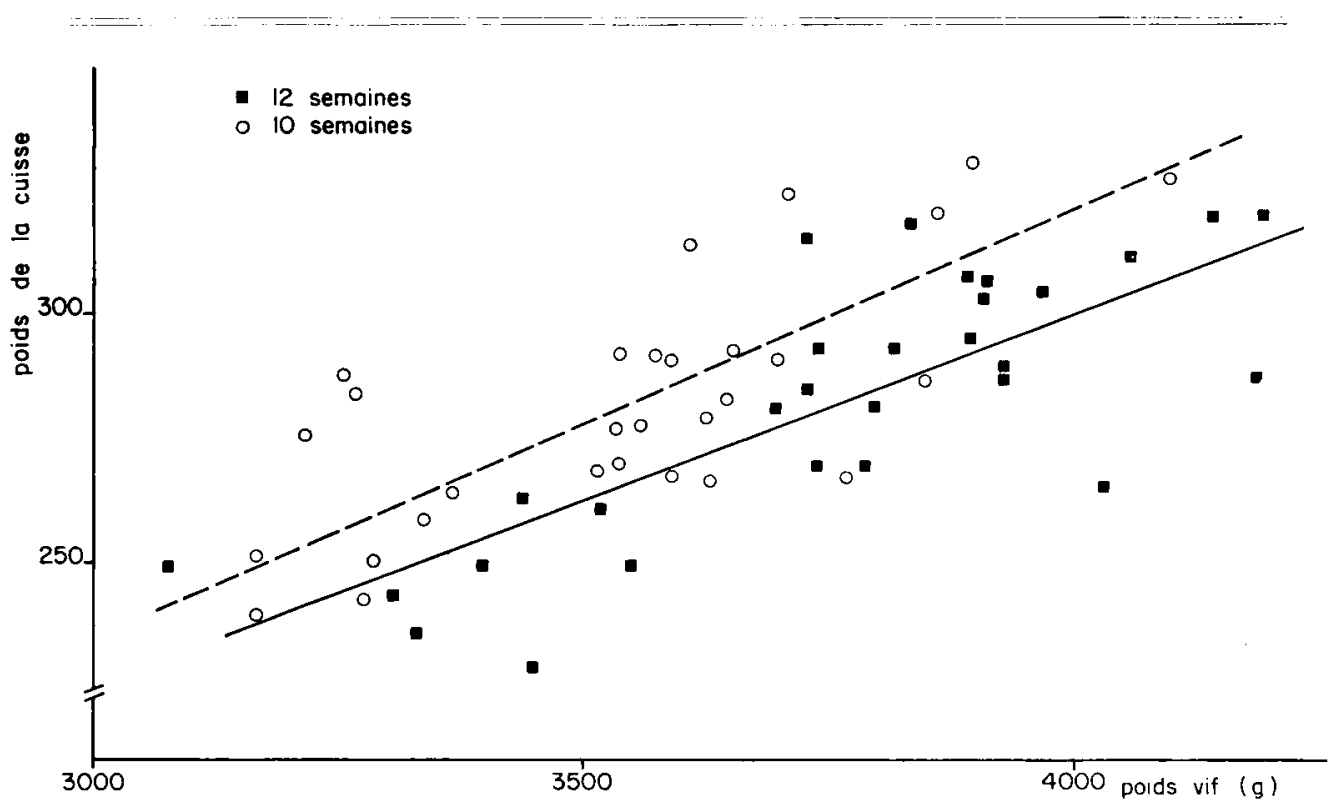

Fic. 2. - Evolution du poids de la cuisse en fonction du poids vif chez le Caneton de Barbarie mâle 
au poids vif lors de ces deux stades d'abattage et qui sont présentées dans la figure $\mathrm{I}$.

Le développement des membres postérieurs est présenté parallèlement à celui des filets dans les tableaux 5, 6 et 7. Pour les lots I4, I5 et I6, il n'y a pas d'effet de l'âge, ni du régime sur la taille de la cuisse. Ians les deux lots carencés, il n'en est pas de niême ; ce qui nous oblige à recourir à l'analyse de covariance. Celle-ci (tab1. 6) révèle que, quel que soit le stade d'abattage, le rapport $\frac{\text { poids de la cuisse }}{\text { poids vif }}$ n'est pas modifié par la teneur en MAT des régimes. Au contraire, l'âge d'abattage réduit significativement le rapport (tabl. 7). Ce phénomène est illustré par la figure 2.

\section{DISCUSSION}

Les résultats exposés précédemment peuvent être discutés d'une part en vue de préciser le besoin en protéines du Caneton, d'autre part pour étudier les principaux facteurs susceptibles d'influencer la qualité de la carcasse.

\section{Le besoin en protéines du Cancton mâle'}

Comme chez la femelle, le besoin en protéines totales du mâle diminue rapidement avec l'âge et atteint des valeurs particulièrement basses pour un oiseau destiné à la consommation. Nos résultats conduisent à penser que ce besoin n'excède pas I 5 p. IOO de protéines de 4 à 8 semaines, et I 2 p. IOO de 8 à Io semaines. Au-delà, il devient inférieur à la plus faible teneur expérimentée dans cet essai. Ie besoin en protéines du Caneton mâle est donc du même ordre que celui de la femelle (LECLERCQ et de Carvilise, I975). Bien que les deux sexes n'aient pas été comparés au cours d'un même essai, le fait de recourir à des conditions expérimentales semblables (origine génétique, mode d'élevage, composition des régimes) rend cette affirmation très probable et en accord avec les conclusions de PII, IA et Quilicr (I973). Cependant, nous proposons une valeur du besoin protéique nettement plus faible pour une croissance beaucoup plus rapide que celle observée par PII.IA et Quir.ICI (I973). La raison doit en être recherchée dans la précision des protocoles utilisés.

\section{L'évolution des caractéristiques anatomiques}

Conme la femelle, le Caneton mâle développe ses masses musculaires tardivement. Sa croissance continue au-delà de l'âge de ro semaines et le gain de poids est alors représenté en majeure partie par des parties comestibles. Si le poids des cuisses demeure constant, les filets ne cessent de croitre en valeur absolue comme en valeur relative. L'animal exigerait alors un apport suffisant de protéines. La croissance pondérale entre Io et I 2 semaines est possible avec un régime renfermant Io,6 p. Ioo de protéines, mais le développement des masses musculaires n'est pas maximum si l'animal reçoit moins de 13,4 p. Ioo de protéines depuis l'âge de 4 semaines. I1 privilégie alors le développement de son squelette et ne parvient pas au cours de cette croissance compensatrice à couvrir totalement les besoins entraînés par l'élaboration de ces masses musculaires. 
Quoi qu'il en soit, nos résultats montrent que c'est l'âge et non le poids qui détermine la proportion de filet. L'âge d'abattage revêt donc chez le Canard une importance particulière pour ce qui concerne la qualité de la carcasse. En outre, si la sélection génétique ne retient comme seul critère que la vitesse de croissance et qu'elle entraîne un abaissement de l'âge d'abattage elle conduira automatiquement à la production de carcasses moins bien pourvues en parties comestibles.

Requ pour publication in décembre 1975 .

\section{SUMMARY}

PROTEIN FEEDING OF MUSCOVY DUCKI,INGS :

REQUIREMENTS IN MALE DUCKLINGS BETWEEN 4 AND I2 WEEKS OF AGE

Hundred male Muscovy ducklings received till the age of 4 weeks the same starter diet largely meeting their requirements (table 1 ). Thereafter the animals were fed with one of the 5 experimental diets. These diets were isoenergetic (12.6 $\mathrm{MJ} / \mathrm{kg}, i . c .3$ ooo Kcal of metabolisable energy per kg and only differed by their protein content: I0.6, I2, I 3.4, I 4.9 and I6.3 p. Ioo (measured values). The groups were called I2, I3, I 4, I5 and 16 according to the theoretical protein content of the diets they received. For each group there were two replicates of ro animals. The ducklings were kept on wire-floor and weighed individually at the ages of 5, 6, 7, 8, 9 and ro weeks. At the age of Jo weeks and 4 days (74 days) half of the animals from each replicate was killed. The other half was weighed at II and I 2 weeks and killed at I 2 weeks and 4 days ( 88 days). After slaughter, carcass yield as well as weights of right breast muscles and thigh were measured.

Table 2 inclicates the live weights at different ages according to the diets ingested. The statistical analysis was made on the individual live weights reported in table 3 . Table 4 shows the results concerning feed intake. Carcass yield and dissection data are given in table 5 .

Table 6 shows the results of the analysis of covariance between breast weight or thigh weight and live weight at each of the two slaughter ages, according to the protein content of the diets. At $7+$ days, the diets did not affect either the proportion of breast of that of thigh. At 88 days, an effect of the diets on the proportion of breast muscles was recorded : groups 12 and I 3 exhibited a significantly smaller proportion of breast.

The analysis of covariance between weight of breast or thigh and live weight according to slaughter age is given in table 7 . Only groups 14 , I 5 and 16 showing identical growths were considered. According to this the proportion of breast significantly increased with age. Conversely, the proportion of thigh decreased with live weight. These findings are illustrated in figures I and 2 .

The protein requirement (diet containing maize and soyabean oil-meal) of male ducklings is not different from that of female ducklings (LECLERCQ and DE CARVILLE, 1975) and does not exceed I5 p. Ioo between $f$ and 8 weeks of age and 12 p. 1 oo between 8 and Io weeks. Beyond that age it becomes inferior to the lowest value tested in the present trial.

\section{RÉFÉRENCES BIBLIOGRAPHIQUES}

Lecherce B., Carville H. de, I975. Besoin en protéines du Caneton de Barbarie entre les âges de 4 et I semaines. Ann. Zootech., 24, 217-227.

PIlla A. MI, Quilici R., I973. Influenza di differenti diete sull'accrescimento e su alcune caratteristiche rilevate alla macellazione delle anatre. II. Anatra muschiata. Ann. Ist. Sper. Zootec., 6, 75-95. 